\title{
MPEDS: Automating the Generation of Protest Event Data
}

\author{
Alex Hanna
}

January 9, 2017

The social media age has drawn vast amounts of attention to modern social movements. Movements such as Black Lives Matter and Occupy Wall Street have reinvigorated discussions about the unequal distribution of income and wealth, the amount of control by multinational corporations and banks, and vast racial disparities in policing, sentencing, and incarceration. As scholarly and public interest in protest increases, there is a growing demand for good data on contentious collective action events in a variety of fields. International relations and foreign policy experts are often interested in using protest event data to forecast political instability and state breakdown. The emerging field of "data journalism" tells narratives about protest activity and political changes around the world. And most relevant for the current project, scholars of social movements and contentious politics need high quality protest event data to understand the emergence, dynamics, and consequences of new social movements and contentious collective action.

However, the lack of high quality protest event data is a chronic issue in social movement research. Comprehensive protest event data with broad spatial and temporal coverage is limited by both the availability of primary sources and speed at which we can code these sources for relevant features for scholarly and practical work. Social scientists have relied primarily on newspapers to gather information about protest events. The biases in using newspapers as primary sources are well-documented by social movement scholars (e.g. Franzosi, 1987; Earl et al., 2004; Ortiz et al., 2005). Biases induced by selective coverage are difficult to address, but incorporating multiple media sources may be an adequate, albeit not perfect, corrective. Given the explosion of electronic archives of newspapers and the availability of new digital media, the potential for identifying protest events is enormous.

With this increasing availability of digital sources from which we can identify protest events, the challenge is to code these sources for collect relevant information. Hand coding newspapers 
has been the traditional strategy for identifying protest events within social movements scholarship (Hutter (2014) provides a recent review of many protest event data and analysis projects). The advantage of this approach is that we can extract a wide range of detailed information from news articles, including types of actions, social movement organizations involved, claims made, size, and whether police or protesters used violence. The main disadvantage of this approach is that it is highly labor-intensive and expensive, requiring careful readings of back issues of daily newspapers or a sample thereof. Because of the high cost, researchers must restrict parameters to the number of newspapers coded, particular geographical regions, and specific time periods. This restriction limits the cases to which we can test hypotheses and the quality of the data in terms of comprehensiveness.

The primary goal of this paper and the larger project which it is initiating is to build, test, and validate an automated system for the coding of protest event data from digitalized news sources, using technological advances from computer science and statistics, namely natural language processing. I call this system the Machine-learning Protest Event Data System, or MPEDS. The aim of MPEDS is to reduce the labor required to generate protest event data and to minimize the biases associated with newspaper coverage of protest events. They will also have reliability rates which are comparable to human coders. The resulting datasets will contain rich information relevant to social movement scholars, include longer-term temporal coverage (including real-time coverage) and introduce the potential of coding for protest events from multiple news sources with worldwide coverage. MPEDS will also be open, available for replication, and extendable by future social movement researchers, and social and computational scientists.

This paper is ordered as follows: I first give a short primer on protest event data and its uses within social movements research. I then introduce the MPEDS, machine learning, and the methodological advances in text as data. I discuss how MPEDS is an improvement over other systems which produce political event data with automated methods. I then outline the components of the MPEDS system - namely the haystack, closed-ended, and open-ended coding tasks. I present evaluation metrics for each part of the system, and in the process compare the suitability of different types of news sources for training the MPEDS classifiers. I then show that many features of MPEDS have comparable reliability to human coders. I close by discussing the future tasks to be accomplished within MPEDS, and suggest implications of the system for social movement research. 


\section{Protest event data}

Protest event data is the "who, what, when, where, why, and how" of collective contentious activity. We want to know who is protesting, what claims they are making, who they are targeting, at what time, in what location, and with what methods of protest. Social movement scholars have used protest event data to study a number of significant phenomenon, including the onset of collective ethnic and nationalist violence (Olzak, 1989, 1992, Beissinger, 2002), protest cycles (Tarrow, 1989), the diffusion of ethnic rioting (Spilerman, 1970, 1971, 1976; Myers, 2000), movement responses to police repression (Khawaja, 1994 ; Earl, Soule and McCarthy, 2003; Earl and Soule, 2006; Davenport, 2010), legislative responses to movement activity (McAdam and $\mathrm{Su}, 2002$ ), and innovation by social movement organizations (Soule, 2009; Wang and Soule, 2012). Within political science, protest event data (as well as data on other political events) is used primarily for political forecasting of political instability (Goldstone et al. 2010) and the onset of political conflict and violence (Brandt, Freeman and Schrodt, 2011, Schrodt, Yonamine and Bagozzi, 2013). Others have highlighted the rise in attention by political scientists towards civil strife, including civil wars, political violence by non-state actors, as well as protest and political expression (Nardulli, Althaus and Hayes, 2015).

Typically scholars have relied on newspapers as records of political and protest events 1 . Within social movements scholarship, protest event data has usually been extracted from newspapers for a specified time period in a single or handful of countries, typically from one or a handful of newspapers at most. Tilly, Tilly and Tilly (1975) coded for violent events in France, Germany, and Italy by using national newspapers over the period of nearly a century. Tarrow $(1989)$ coded on collective protest in Italy's main newspaper of record from 1966-1973. Olzak (1992) coded for ethnic collective events in the United States from the New York Times from 1877-1914. In their study of "new social movements", Kriesi et al. (1995) coded for protest events from 1975-1990 in four European countries from four newspapers.

Most of these datasets have been collected to support their authors' specific research projects and are thus rarely re-analyzed by other scholars. However, recently scholars have made an effort both

\footnotetext{
${ }^{1}$ Official sources such as government and police records are not kept consistently, are contingent on the willingness of a government in sharing their data and how readily accessible those data are, and often don't contain the information which movement scholars are interested. For this reason, only a few datasets have been assembled (Maney and Oliver (2001) for Madison, Wisconsin; McCarthy, McPhail and Smith (1996) for Washington, DC; and Chris Sullivan and Christian Davenport's work on Guatemala).
} 
to establish a standard methodology for collecting event data and to collect more comprehensive data to be deployed in a variety of movement research. In an effort to establish a common (i.e. not project-specific) method for the collection of event data, Franzosi (2004, 2010) outlines "quantitative narrative analysis," which consists of a formal grammar for documenting historical narratives. Within this grammar, coders must identify the subject, object, and action of an event from historical sources, including newspapers.

Many of these datasets use a handful of news sources, and there is a large body of literature which highlights the differences between news sources nominally covering the same time periods and geographic areas (Franzosi, 1987; Earl et al., 2004). Although there is no perfect measurement of the underlying flow of collective events to provide a basis for comparison, some have suggested that compiling events from many different news sources that vary in location and political slant is the best way to get as close as possible to the true flow of events (Woolley, 2000; Myers and Caniglia, 2004). For example, in his study of collective protest and violence in former Soviet states, Beissinger (2002) uses a wide mix of Western, official Soviet and post-Soviet, and émigré news sources, many of which he obtained from news clipping archives that had been compiled by other:2. Similarly, Carter (1983) compiled a comprehensive dataset of urban riots between 1964 and 1971 in the US from multiple sources, including the Congressional Quarterly's Civil Disorder Chronology, the New York Times, and the Washington Post. The rise of electronic archives of newspapers and the availability of new digital media have made it even easier to access multiple news sources.

\section{The Machine-Learning Protest Event Data System}

The goal of this paper is to introduce and highlight the advantages of my own system, the MachineLearning Protest Event Data System, or MPEDS. The goal of MPEDS is to provide high quality protest event data using tools from machine learning and natural language processing with little to no human intervention. Before introducing this system, I briefly highlight the growing field of machine learning and data science, and the methods which it introduces. I then review similar automated systems for political event data generation and note how MPEDS improves upon these

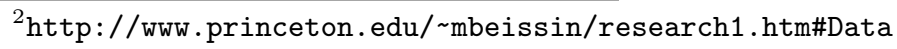


systems.

\subsection{Machine Learning and Text as Data}

Machine learning can be defined as a set of probabilistic methods that can automatically detect patterns in data and use that information to make predictions in other data (Murphy, 2012). Machine learning methods are often used for classification, ranking, or recommendation. Examples of each include deciding whether Twitter users are liberal or conservative based on their tweets (classification, e.g. Conover et al., 2011), Google's "Priority Inbox" (ranking), and Netflix's suggestions of new products for consumption (recommendation).

Machine learning has become ubiquitous in applications within computer science, and familiarity with its principles and methods is a prerequisite in the burgeoning field of "data science." However, it is only beginning to make inroads within the social sciences, primarily within the field of natural language processing or what has come to be known as "text as data" within political science and digital humanities. The intersection of machine learning and natural language processing has been a fruitful one and has produced a set of common methods and procedures. Within social science, Grimmer and Stewart (2013) provide a good overview of different modes of machine learning, procedures required for treating text as data, and applications within political science. The cultural analysis journal Poetics dedicated an issue to topic modeling, a form of unsupervised learning for text, and discusses its implications for social sciences (Mohr and Bogdanov, 2013).

Machine-assisted approaches to political event data have been in use for nearly 30 years, since the inception of the Kansas Event Data System (KEDS; Gerner and Schrodt, 1994) and its progeny (PETRARCH/Phoenix; Schrodt, Beieler and Idris, 2014). More recently, there have been several approaches which incorporate machine learning methods into their pipelines. The SPEED system (Nardulli, Althaus and Hayes, 2015) uses supervised machine learning to help filter out articles which do not contain an event of interest. Croicu and Weidmann (2015) use an ensemble of supervised machine learning classifiers to filter out irrelevant articles in a similar manner to the SPEED project. Neither of these projects, however, attempts to construct a fully automated system. The most significant attempt for a full automated process has been attempted by the Political Conflict in Europe in the Shadow of the the Great Recession (POLCON) project ${ }^{3}$. Wueest,

\footnotetext{
${ }^{3}$ http://www . eui . eu/Projects/POLCON/Home.aspx
} 
Rothenhäusler and Hutter (2013) and Marakov et al. (2015) have attempted an initial foray into full automated but had limited success. Many of the issues they faced are endemic in this full automation of protest event data extraction, which I will outline further below.

\subsection{Comparing MPEDS to Other Approaches}

MPEDS differs from other automated approaches to producing protest event data in several ways. Like SPEED, it uses a supervised method, meaning humans provide "training" data on which its classifiers are based. And like KEDS, it aims to be open and transparent in its data production and pipeline. However, MPEDS differs from other automated event data projects in two major regards: scope of the event and amount of data provided for each event.

Instead of attempting to do many things somewhat well, MPEDS attempts to do one thing very well: identify protest events. In other automated projects, the protest event is ill-defined or subsumed under a more general political event. This has the consequence of both providing a very sparse amount of information for any given protest event (since all political interactions are reduced to a common denominator of information) and by shifting the definition of a protest event such that it fits more neatly into other kinds of political interactions, which has the consequence of forcing the event into a state-centric idea of political interaction. KEDS and its progeny fall victim to the data sparsity problem. Every event is a dyad between two state or non-state actors. Beyond defining actors, targets, and a single political action, no other information is provided about the event. The CAMEO ontology and the SPEED system define protest in a manner that is a poor fit for movements research. The CAMEO ontology used with KEDS is geared towards international relations events - namely, mediation - and not social movement ones (Schrodt, Beieler and Idris, 2014). In addition, CAMEO's event ontology was originally developed to document actions in the Middle East, thus may be skewed in ways that restrict its applicability to other regions. SPEED defines a protest event as an act of "political expression", which includes many of the categories considered as protest by movement scholars, but also includes other behavior, including the publication of dissident media and cultural arts (cartoons, movies, plays).

MPEDS defines a protest event based on an engagement with social movement theory and a survey of hand-coded datasets within the social movement literature. It also differentiates itself from other automated projects by attempting to find a good medium between the sparse dyadic data of 
KEDS and the hand-coded and textured data produced by a hand-coded project. MPEDS provides a number of variables on protest events which have been of historical importance to movement scholars. The system is structured in this way such that a more fully automated solution can process news sources with minimal human intervention. Lastly, the MPEDS system, the human coder web interface, and the data produced by MPEDS will be offered as open-source and distributed publicly. In addition, events will include an audit trail, such as a URL (if available), and the article title and news source, such users can identify the source text of the article and reassess the data on a qualitative basis. MPEDS is thus oriented to produce data which is primarily of interest to movement scholars, both by definition of the event and by the information which is included in each record.

\subsection{MPEDS Architecture}

Within MPEDS, there are three discrete tasks: the haystack task, the closed-ended coding task, and the open-ended coding task. The haystack task discerns whether a document mentions a protest event. I call this the haystack task because the problem is largely imbalanced - articles that mention protest are rare relative to the total number of articles in any given news source. The closed-ended task attempts to classify several variables which can take on a discrete number of values. I focus on three variables: the dominant form of the protest, the main target of the protest, and the main issue of the protesters. The task is, for each of the documents identified as mentioning a protest event, is to classify the document for each of these variables. The final task is to pull out relevant information of the protest, the "open-ended coding" task. I focus on a protest's size, its location, and the name(s) of social movement organizations involved (if any).

The first two tasks can be treated as multiclass document classification problem; the last task can be treated as a named entity recognition and pattern matching task. Table 1 summarizes the tasks and the methods of data generation. These tasks will be outlined in detail below.

The MPEDS project is also collecting news text and coding data by hand in order for the system to use as a training data. Using a web interface, coders must first discern whether an article contains a protest event (the haystack task) and then highlight the text in which variables

of interest are present. Although many of the variables (e.g. claims) are not explicit in the text, we must rely on the text itself to produce variables of interest. After this "first pass" of coding, articles 


\begin{tabular}{lll}
\hline Variable & Task & Method \\
\hline Contains protest? & Haystack & Binary classification \\
Issue & Closed-ended coding & Multiclass classification \\
Form & Closed-ended coding & Multiclass classification \\
Target & Closed-ended coding & Multiclass classification \\
Size & Open-ended coding & Pattern matching \\
Location & Open-ended coding & NER + Dictionaries (gazetter) \\
Organization names & Open-ended coding & NER + Dictionaries \\
\hline
\end{tabular}

Table 1: Variables and methods of classification

which are candidates for event coding are passed to a "second pass", in which coders disentangle multiple events in a single article, categorize forms, claims, and targets into discrete categories, and double-check the coding for specific locations, dates, social movement organizations, and crowd sizes.

The main aim in creating this hand-coded dataset is not comprehensiveness of coverage over a particular time period or particular news source. The goal is incorporating enough protest articles from a diverse number of sources in order to account for all the different ways in which a news source may talk about protest activity. Different sources possibly use different words and word combinations to talk about a protest event. Therefore, we code for news sources which may have stylistic differences in reporting, rather than simply spatial variation. Figure 1 illustrates the entire MPEDS pipeline, including the process of incorporating new training data. The methodology is as follows:

1. Select a number of news sources of interest. Include variation based on location, audience (national, regional, international), format (newspaper, news wire), and time period in order to account for any period effects in language.

2. Sample an adequate amount of articles to generate sufficient protest articles for building training and test sets. Given prior testing and other machine learning projects (e.g. Hopkins and King, 2010), this is between 50 to 100.

3. Search a news database (e.g. Lexis-Nexis) with a broad search term that includes all uses of protest-related words. This helps to reduce extraneous articles.

4. Pipe these articles to a first pass of coding in which coders decide whether an article involves a protest or not, and highlight relevant parts of the text. This task filters out over $80 \%$ of articles.

5. Send all articles which are labeled as protest to a second pass in which coders construct discrete events from articles. 


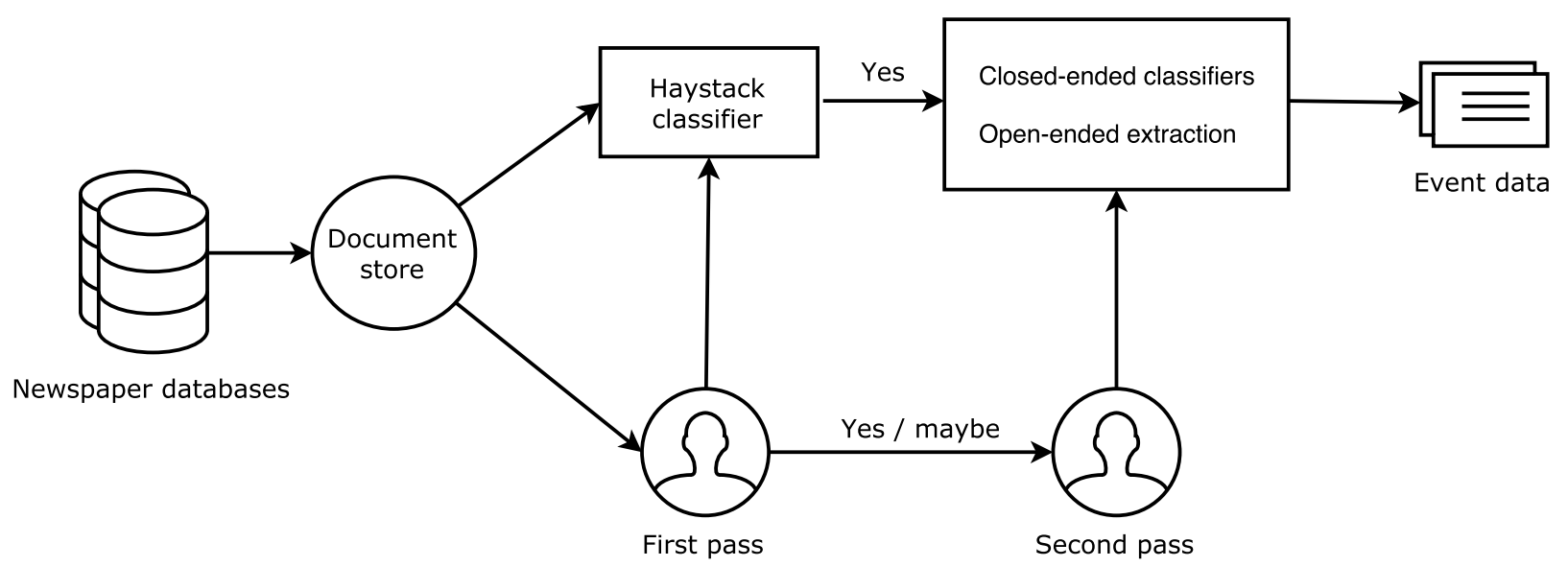

Figure 1: MPEDS pipeline with training.

\subsection{Potential Training Data: Dynamics of Collective Action}

MPEDS originally attempted to use an existing protest dataset as training data. The Dynamics of Collective Action (DoCA) dataset is, to date, the largest protest event dataset of events occurring in the United States 4 . To generate this dataset, humans hand coded articles from the New York Times from 1960 to 1995. This resulted in a dataset of nearly 21 thousand unique events. DoCA includes any event which meet the following criteria: (1) collective acts; (2) public actions; (3) protest actions (e.g. not a fundraising event or a closed group meeting); and (4) are making a specific claim or grievance about the desirability to change society 5 . In addition to coding for protest, DoCA includes ethnic/racial conflict events and lawsuits related to social movement activity. However, in order to have a more strict specific definition of a protest event, we excluded these events from our analysis. Each event is coded for a comprehensive list of variables, including date, size, location, a qualitative description of participants and events, claims, forms of protest, protest targets, initiating groups involved, presence of violence, and presence of police.

I treat DoCA as a potential training set for the haystack task. DoCA seems well-suited for this purpose, given the large number of events in the dataset and the number of events which can be matched to their original articles in the New York Times. To match events to source articles, I use the New York Times Annotated Corpus ${ }^{6}$ obtained by the University of Pennsylvania Linguistic

\footnotetext{
${ }^{4}$ The dataset and accompanying codebooks can be found at http://www.stanford.edu/group/ collectiveaction/cgi-bin/drupal/node/1.

http://www.stanford.edu/group/collectiveaction/BRIEF\%20EVENT\%20GUIDE. docx

6 https://catalog.ldc.upenn.edu/LDC2008T19
} 
Data Consortium (LDC-NYT), a machine-readable dataset of 1.8 million New York Times articles from 1987 to 2007. DoCA contains a total of 3,570 contentious collective action events during this period that should have a corresponding article in LDC-NYT, that is, from 1987 to 1995 . In practice, however, I have found that not all records in DoCA could be matched to a source article in LDC-NYT, either due to a malformed transcription into LDC-NYT, DoCA coders sourcing the event from an AP wire report that does not appear in LDC-NYT, or some updated or otherwise changed title in LDC-NYT. However, with minor data cleaning I matched about 88\% (3,214 of $3,570)$ of the articles in DoCA to their source texts.

From 1987 to 1995, there were nearly 820 thousand articles in the New York Times, while there are only about 400-500 events per year within DoCA. Following Leetaru and Schrodt (2013), I filtered out a handful of common titles related to business, finance, and sports (e.g. "Business Report") which are not be relevant to the project. The LDC-NYT also contains a field which lists a taxonomical classification when indexed online, and of this I exclude Business, Finance, Sports, and Classified categories. I also exclude Weddings and Book Reviews based on the New York Times index field. This filters out more than half of the articles which we can assume do not mention protests. For the final filter, I sampled all articles from the LDC-NYT on each date in which there was a record in DoCA using a broad search string, described in Appendix B. In our final count, we have just over 50,000 potential protest-related articles.

\subsection{Data generated from the MPEDS project}

The MPEDS project has collected news text data from over a dozen sources, including several local and national US newspapers, and news wire services. I focus on several sources across all geographical coverage areas. Each source is displayed in table 2, along with the number of articles used in its training set, the number of articles found to contain a protest by human coders, and that value as a percentage of total articles. All sources (except for DoCA, which runs from 1987-1995, and NYT, which is from 1996-2007) were sampled from the beginning of 1995 to the end of 2010. We used the search string specified in Appendix B to filter out articles. From each source, we drew a sample of 150 dates which was stratified to oversample on Sunday editions. National news sources include the New York Times, the Washington Post, and USA TODAY, local sources include the Austin American-Statesman, Omaha World Herald, and the Atlanta Journal-Constitution, and 
news wires include Agence France-Presse and the Associated Press. The New York Times data were drawn from the LDC-NYT dataset and news wires from the Annotated English Gigaword dataset 7 also provided by the Linguistic Data Consortium. The other sources were downloaded from Lexis-Nexis. Following Nardulli, Althaus and Hayes (2015), I stored all articles and related metadata in an Apache Solr document store for quick access, version control, and indexing.

MPEDS defines a protest event in the following manner: the event must involve some form of claims-making and grievance expression, have sufficient information for coding, i.e. location and date, occur in public, and include at least some non-institutional actor. A full definition of acceptable (and non-acceptable) protest events is located in Appendix A. Coders went through at least one month of training which included weekly team discussions and reviews of reliability reports generated from the project data. I included as a protest any article in which over $50 \%$ of coders labeled as such.

Table 2 reports the number of protest articles in each data source as a percentage of the total sample. DoCA has the lowest number of $2.15 \%$ and NYT has fourth lowest at $5.31 \%$. Theoretically, these two values should be the same. This either indicates underreporting by DoCA coders, overreporting by MPEDS coders, or a significant change in the rate of reporting protest events between the 1987-1995 and 1996-2007 period. Each local source (ATL, OMA, AUS) has a protest article occurrence of less than $6 \%$. WPO and USA, the other national newspapers, have rates of protest articles of $9.73 \%$ and $9.3 \%$, respectively. The news wire services report the highest percentage of protest articles, $12.41 \%$ for the APW and $16.41 \%$ for the AFP.

\section{$3 \quad$ Haystack coding}

The haystack task itself proved to be one of the most difficult parts to train, tune, and to validate of the whole of MPEDS. It's of little surprise that many of the attempts to automate the creation of protest event data have stopped after carefully tuning a set of classifiers or dictionary rules which are able to adequate capture a good deal of the events of interest. This is due to the fact that the social object of the "protest" is itself heterogeneous, difficult to define, and requires explicit boundaries to separate it from routine crime, sport hooliganism, terrorism, or other forms of political

\footnotetext{
7 https://catalog.1dc.upenn.edu/LDC2012T21
} 


\begin{tabular}{lrrr}
\hline Source & Total & Protest & Protest \% \\
\hline Agence France-Presse (AFP) & 4131 & 678 & 16.41 \\
Associated Press Worldstream (APW) & 3891 & 483 & 12.41 \\
The Washington Post (WPO) & 3413 & 332 & 9.73 \\
USA TODAY (USA) & 903 & 84 & 9.3 \\
Austin American-Statesman (AUS) & 663 & 38 & 5.73 \\
The New York Times (NYT) & 3051 & 162 & 5.31 \\
Omaha World Herald (OMA) & 784 & 32 & 4.08 \\
The Atlanta Journal and Constitution (ATL) & 1350 & 55 & 4.07 \\
Dynamics of Collective Action (DoCA) & 50266 & 1079 & 2.15 \\
\hline
\end{tabular}

Table 2: Descriptive statistics on news sources for training datasets. Name abbreviations are in parentheses.

violence. Indeed, Hutter (2014) notes how the definitions of protests seem to shift with the focus of the researcher or the specific project. The MPEDS project has sought to be question-agnostic in our own definition, but this naturally does not prevent any of our own intellectual and personal preoccupations from slipping into the analysis. In this section, I outline the steps taken towards developing the haystack task, including text preprocessing, selecting sources, and evaluation.

\subsection{Preprocessing and evaluation}

Article texts went through a series of pre-processing procedures before being used in the machine learning system. They were converted to lowercase and stripped of punctuation and stop words (e.g. common connecting words like "the", "a"). I converted words in the article to numerical representation (a series of feature vectors in machine learning terms) using the term frequencyinverse document frequency metric, or tf-idf. This metric is a measure of word prevalence for word $i\left(w_{i}\right)$; it is calculated by number of times $w_{i}$ appears in a document divided by the number of times $w_{i}$ appears in the whole corpus of documents.

I evaluate the accuracy of the system by using metrics of precision and recall from the machine learning literature. These metrics are based on the number of true positives (TP), or correctly classified documents, compared to those which are false positives (FP) or false negatives (FN). Precision can be defined as the fraction of documents correctly classified from the set of all the documents in the class of interest (Equation 1), while recall can be defined to the fraction of documents correctly classified from the set of all documents (Equation 2). Maximum precision 
would indicate the absence of false positives, while maximum recall would indicate the absence of false negatives. Precision and recall are thus analogous to the Type I (incorrect rejection of a true null hypothesis) and Type II errors (failure to reject a false null hypothesis), respectively. Precision and recall are tradeoffs by definition.

$$
\begin{gathered}
\text { precision }=\frac{T P}{(T P+F P)} \\
\text { recall }=\frac{T P}{(T P+F N)}
\end{gathered}
$$

I used $F_{\beta}$-scores (or F-score, Equation 3 ) to evaluate the overall model. This score is the harmonic mean of the recall and precision. In the haystack task, I use the $F_{2}$-score, which weights the recall with more importance. Otherwise, I use the $F_{1}$ score, which weights them equally.

$$
F_{\beta}=\left(1+\beta^{2}\right) * \frac{\text { precision } * \text { recall }}{\left(\beta^{2} * \text { precision }\right)+\text { recall }}
$$

Within the machine learning literature, there are no hard numerical cutoffs on the acceptability of any one of these metrics. The cutoff is more or less application-dependent. If a researcher is more interested in retrieving documents of interest with some level of noise, prioritizing recall should be more important. Conversely, if the researcher wants to identify the most relevant documents and risk losing some in the process, precision should be prioritized. For this paper, I use 0.65 as a lower boundary for an acceptable $F_{\beta}$-score.

Classifiers were tested using $\mathrm{k}$-fold cross-validation with $k=3$. K-fold cross-validation withholds a single slice or "fold" of the data for testing while training on the other $k-1$ folds. For the haystack classification, I used an ensemble classifier, which has been used with success in other political and event analysis (e.g. Grimmer, Messing and Westwood, 2014, Croicu and Weidmann, 2015). Ensemble methods work by applying several different classifiers to the same dataset and giving each classifier a "vote" on the article's classification. After testing several combinations, I obtained the best results combining a support vector machine (SVM) classifier with a linear kernel, a logistic regression (LR) classifier, and three stochastic gradient descent (SGD) classifiers with different loss functions: the hinge loss function, the perceptron loss function, and the Huber loss 


\begin{tabular}{llrlrlr}
\hline Source & All-P & Own-P & All-R & Own-R & All-F2 & Own-F2 \\
\hline afp & 0.56 & 0.67 & 0.84 & 0.63 & 0.76 & 0.64 \\
apw & 0.51 & 0.74 & 0.88 & 0.68 & 0.77 & 0.69 \\
atl & 0.58 & 0.35 & 0.54 & 0.54 & 0.55 & 0.49 \\
aus & 0.67 & 0.41 & 0.73 & 0.57 & 0.72 & 0.52 \\
doca & - & 0.33 & - & 0.58 & - & 0.50 \\
nyt & 0.58 & 0.54 & 0.61 & 0.53 & 0.61 & 0.53 \\
oma & 0.60 & 0.20 & 0.47 & 0.34 & 0.49 & 0.29 \\
usa & 0.45 & 0.35 & 0.55 & 0.49 & 0.52 & 0.45 \\
wpo & 0.57 & 0.65 & 0.60 & 0.42 & 0.59 & 0.45 \\
\hline
\end{tabular}

Table 3: $F_{2}$ score per test source and each training source. Own-* is the metric using only the same source in training. All-* is the metric using all sources in training.

function. For features, I used the tf-idf metric on unigrams and bigrams, that is, one- and two-word combinations. I discuss alternative model specifications and feature selection below.

In practice, multiple sources would be used train the haystack classifier, rather than just one. This is because we want to be able to capture events in a variety of sources, not just one or two major ones. To this end, I first assess classifier performance based on a training set composed of the same source as the test set. I then move to evaluation of classifiers based on every combination of two sources, and conclude with classifiers based on all sources.

\subsection{Results}

Results from the haystack task are reported in Table 3 , which reports the precision, recall, and $F_{2}$ scores for the classifiers using its own source and all sources. Since there is only one combination of training choices for both the classifier based on all sources or its own source, only those two are reported. Figure 2 plots the distribution of $F_{2}$ scores by classifiers trained on own source, pairs of sources, and all sources. For DoCA, I only evaluate the classifier based on its own articles. I do this to illustrate the adequacy of using DoCA itself as a training set.

The most notable things from Table 3 seems to be, first, the large disparity between different sources, and second, the large gains when using the classifier trained on all sources. Results for the own classifier range from 0.29 to 0.69 . The classifiers which perform the worst are the local Omaha paper (0.29), then two national sources - the Washington Post (0.45) and USA TODAY (0.45). While the low accuracy for the Omaha paper could be attributed to the small amount of training 


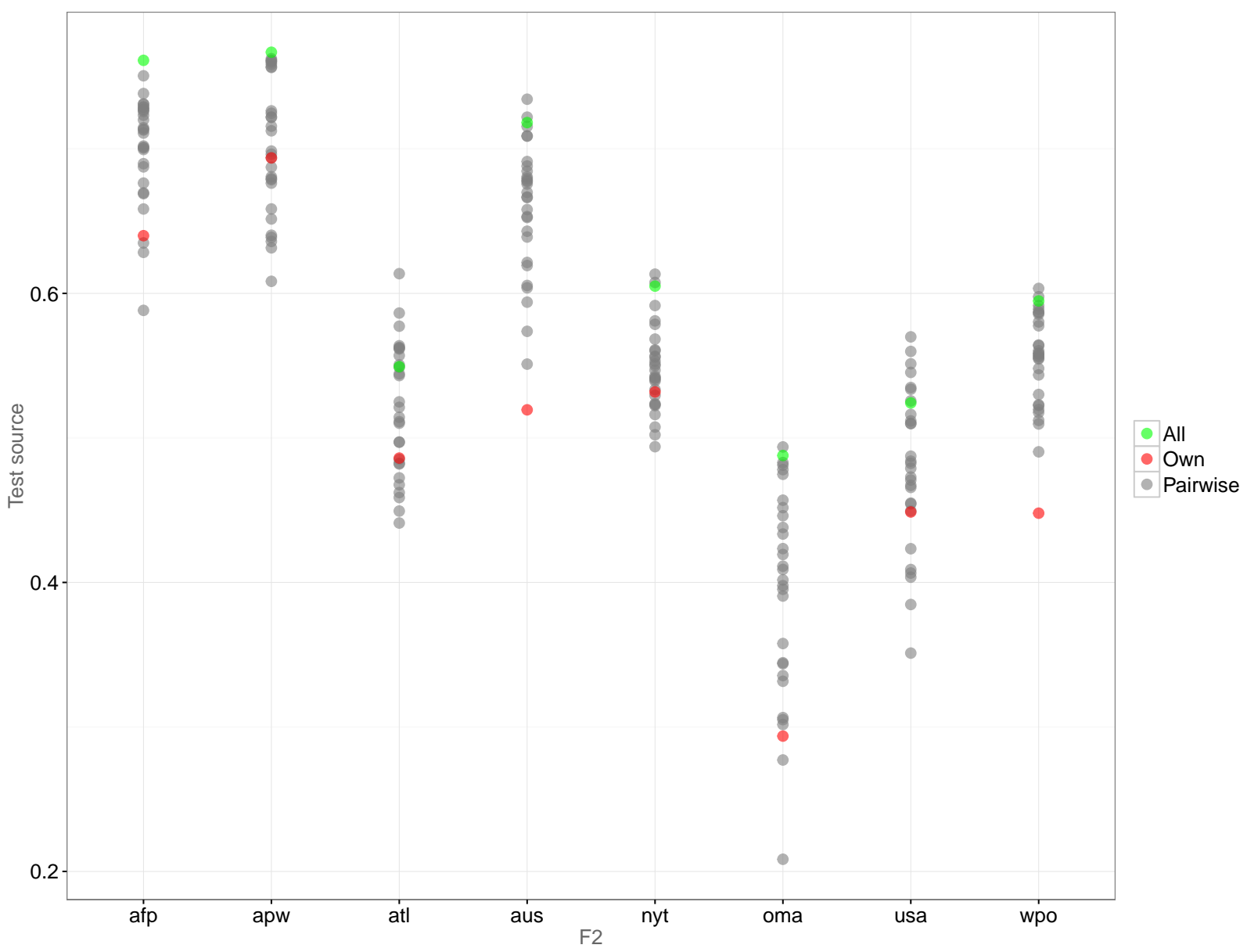

Figure 2: $F_{2}$ scores for classifiers trained on all, pairs, and their own sources

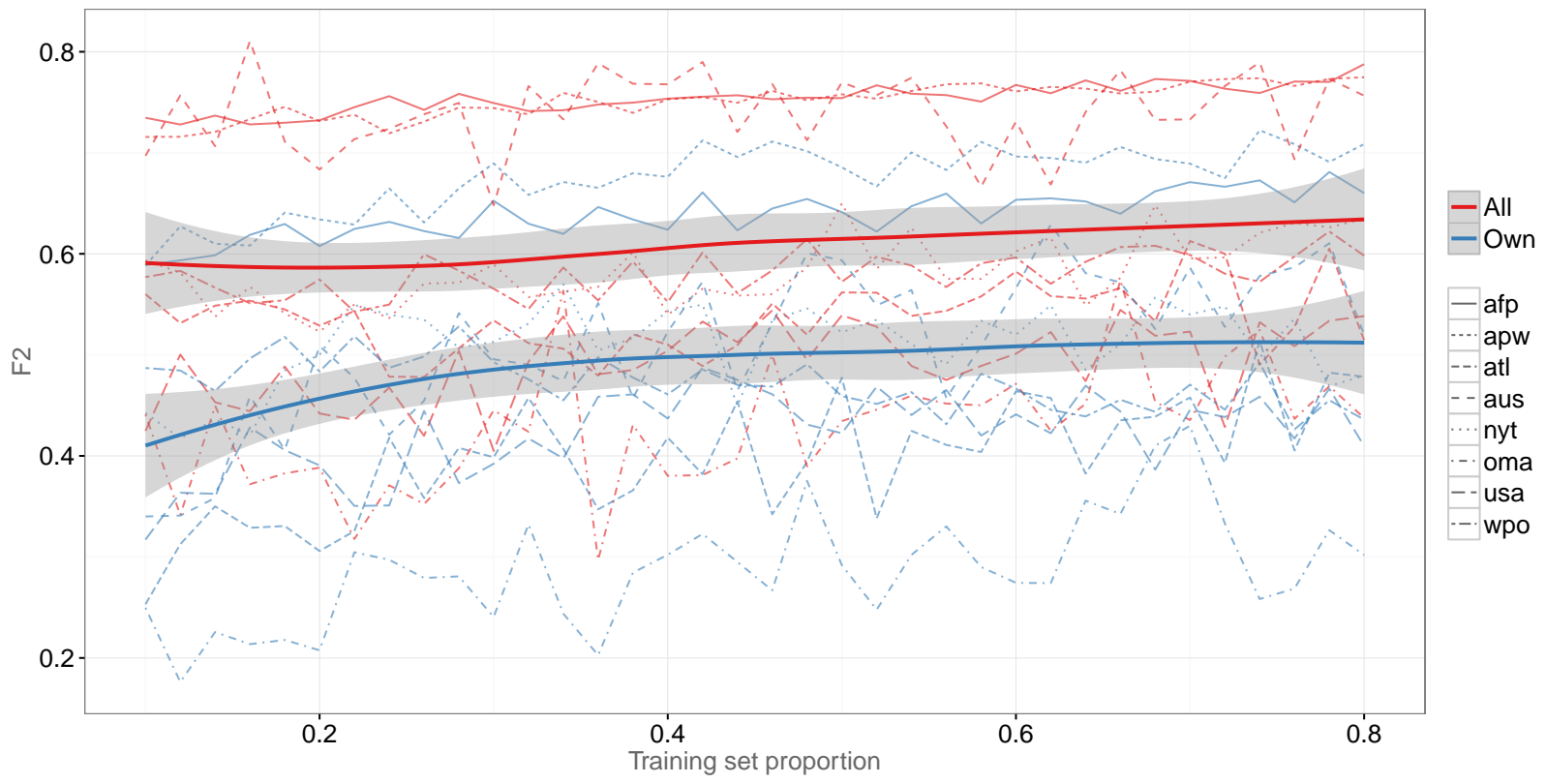

Figure 3: $F_{2}$ scores for own and all sources, by training proportion size 
data used in training, the larger national papers do not have that issue. The best performing own classifiers are the news wire services, AFP (0.64) and APW (0.69). In the middle is the DoCA (0.50) and the New York Times (0.53). Their F-scores are very similar, but there is a large gap between their precision (DoCA's 0.33 compared to NYT's 0.54) and recall (DoCA's 0.58 compared to NYT's 0.53). This would seem to indicate that there is a large number of false positives which are being reported from DoCA. These results merit a small note.

In a separate analysis, I sampled 47 articles which had been marked as false positives by the classifier trained on DoCA data. Of these 50 articles, 31 of them were "false false positives", that is, they were shown to be articles that should have been in DoCA by the project's own criteria but were not. This result highlights a rather large margin of error in DoCA, introduced either by coder fatigue or technological error.

The incorporation of more training sources seems to universally provide for better accuracy. In Figure 2, the gray points represent F-scores for classifiers trained on pairs of sources. In a few cases, these classifiers decrease accuracy, especially with the NYT, APW, and USA sources. This typically is the case when its own source is not part of the pairwise source. But on the whole, the pairwise comparisons provide a net positive.

In nearly all cases, incorporating all sources provides for the best or one of the best classifiers, noted by the green points. All classifiers see an increase in $F_{2}$ score. The largest gains are seen by two local papers - AUS and OMA. Each source sees at least an increase of 0.06 in F-score. The floor for accuracy is now 0.49 (OMA) and the maximum is 0.77 (APW). The addition of more sources therefore provides more heterogeneity in reporting and increases classifier power by a large factor.

It's worth nothing that the news wire services have the best accuracy of any of the news sources. News wires have the highest proportion of protest articles in the dataset. If one is interested in capturing the most events on a worldwide basis - rather than detecting events which are "socially significant" - then it seems like news wires would be a good place to search. Indeed, other event data researchers have noted the virtues of news wire services as well, despite their other drawbacks (Schrodt, Davis and Weddle, 1994; Schrodt, Simpson and Gerner, 2001).

Figure 3 reports the increase in $F_{2}$ score as more of the training set is used for training. The solid line is a LOESS regression across all news sources tested. There is a consistent pattern of most sources which use all training sources for classifier as having better accuracy. On average, 
even a training set using $20 \%$ of data for training still does better on the whole than using $80 \%$ of data for training for the individually-sourced classifiers.

While the haystack task seems straightforward from the outset, since it is a simple matter of detecting whether an article mentions a protest or not, the task is more complicated than it seems at first glance. The variation in accuracy with a similar classifier across multiple news sources seems to point to some kind of fundamental aspect of the news text which impedes a simple binary classifier. Using multiple news sources with an ensemble of classifiers seems to be the best strategy.

In terms of feature selection, I chose to use a simple bag-of-words approach. This approach is computationally inexpensive and can be accomplished with many well-defined tools. However, it may be possible to use part-of-speech tags of words to discern between different uses of words. For instance, there are the semantic differences between $\operatorname{March}_{N N P}$ (the month), $\operatorname{march}_{N N}$ (the noun and actively moving group of people), and march $_{V B Z}$ (the 3rd person singular present verb and common protest activity) 8 . There have also been other great advances in word sense disambiguation with the release of the word2vec too 9 , which is very good at finding similar words based on word context and constructing analogies between sets of words. Both part-of-speed tagging and word2vec are more computationally intensive in the preprocessing stage, however.

One solution I attempted was reducing the dimensionality of the feature space using Latent Dirichlet Allocation (Blei, Ng and Jordan, 2003). Latent Dirichlet Allocation (LDA or topic modeling) is a hierarchical Bayesian model which allows documents to belong to multiple classes (or topics). Each word in the document has a distribution over the topics and is thus more flexible than supervised classifiers. However, this dimensionality reduction approach did not yield better results for the haystack task. There may be ways to successfully apply other unsupervised methods to the haystack classification task, but with the combination of several sources the results are sufficient for our purposes.

\footnotetext{
${ }^{8}$ Subscripts are adopted from the Penn Treebank's part-of-speech tags: https://www.ling.upenn.edu/courses/ Fall_2003/ling001/penn_treebank_pos.html

https://code.google.com/p/word2vec/
} 


\section{Closed-ended coding}

For the closed-ended coding task, I created classifiers for each of the variables which take discrete values: target, issues, and forms. As noted by the Figure 1, the training values were sourced by second-pass coders, and most articles were coded at least twice in the process. Coders could assign more than one value to an article and therefore compound variables constitute their own class. For instance, rallies/demonstrations and marches tend to have a high rate of co-occurrence, so one frequently used compound value for the form variable is "Rally/demonstration-March". I limited the cardinality of these compounds to two, given that there is a very long tail on possible combinations. A value was also not used in the cross-validation set if it did not appear at least than 30 times.

In order to decide which values to use, I constructed a set of coding rules for inclusion of training data values.

1. Total agreement: If there was total agreement, i.e. every value is the same for all coders, then use all values as expected.

2. Partial agreement: This is if coders agreed on one or more values but not others. Two cases apply here: if there are more than two coders and there are values which have taken on a majority vote, use the majority vote (e.g. coder 1: march, coder 2: march, coder 3: rally, use march). Otherwise, use the intersection of all coders.

3. None vs. any: One coder hasn't coded a value or has coded None of the above, while the other coder has. Use the non-none value.

4. Total disagreement: In the last case, coders do not agree on anything. Discard the case and do not use it in the analysis.

After testing several different classifiers for each variable, I settled on different classifiers for each variable. For form, I used the LR classifier, for issue, an SGD classifier, and for target, an ensemble voting classifier based on SVM, SGD, and LR. Each classifier used a "One vs. the Rest" approach, in which a separate classifier was trained for each value such that the classification task assessed fit for that particular value versus all other values.

Like in the haystack task, I use a 3-fold cross-validation method to assess the classifier accuracy. Tables 4 . 5, and 6 report the precision, recall, $F_{1}$, and number of cases across all folds for each of the closed-ended variables. I chose to use the $F_{1}$-score for this task because there is no theoretical reason in which recall should be more important to precision in this task. Tables 7, 8, and 9 report 


\begin{tabular}{lrrrr}
\hline & $\mathrm{P}$ & $\mathrm{R}$ & $\mathrm{F} 1$ & $\mathrm{~N}$ \\
\hline 0: Blockade/slowdown/disruption & 0.58 & 0.07 & 0.11 & 46 \\
1: Boycott & 0.87 & 0.35 & 0.50 & 54 \\
2: Hunger Strike & 0.95 & 0.60 & 0.73 & 53 \\
3: March & 0.64 & 0.38 & 0.47 & 191 \\
4: Occupation/sit-in & 0.50 & 0.06 & 0.10 & 35 \\
5: Rally/demonstration & 0.66 & 0.93 & 0.77 & 809 \\
6: Rally/demonstration-March & 0.33 & 0.11 & 0.16 & 72 \\
7: Riot & 0.63 & 0.34 & 0.43 & 82 \\
8: Strike/walkout/lockout & 0.84 & 0.85 & 0.85 & 273 \\
9: Symbolic display/symbolic action & 0.59 & 0.17 & 0.26 & 52 \\
10: _none_ & 0.00 & 0.00 & 0.00 & 13 \\
\hline
\end{tabular}

Table 4: Form accuracy metrics

\begin{tabular}{lrrrr}
\hline & $\mathrm{P}$ & $\mathrm{R}$ & $\mathrm{F} 1$ & $\mathrm{~N}$ \\
\hline 0: Abortion & 0.94 & 1.00 & 0.97 & 30 \\
1: Anti-colonial/political independence & 0.48 & 0.52 & 0.50 & 67 \\
2: Anti-war/peace & 0.61 & 0.70 & 0.65 & 132 \\
3: Civic violence & 0.54 & 0.21 & 0.30 & 43 \\
4: Criminal justice system & 0.64 & 0.57 & 0.60 & 96 \\
5: Democratization & 0.65 & 0.73 & 0.68 & 172 \\
6: Economy/inequality & 0.61 & 0.55 & 0.58 & 130 \\
7: Environmental & 0.71 & 0.91 & 0.79 & 64 \\
8: Foreign policy & 0.53 & 0.37 & 0.42 & 82 \\
9: Human and civil rights & 0.52 & 0.27 & 0.35 & 78 \\
10: Immigration & 0.81 & 0.81 & 0.81 & 37 \\
11: Labor \& work & 0.80 & 0.92 & 0.85 & 341 \\
12: Political corruption/malfeasance & 0.52 & 0.48 & 0.49 & 94 \\
13: Racial/ethnic rights & 0.50 & 0.60 & 0.53 & 81 \\
14: Religion & 0.82 & 0.86 & 0.84 & 115 \\
15: Social services \& welfare & 0.49 & 0.39 & 0.41 & 52 \\
16: none_ & 0.31 & 0.16 & 0.21 & 85 \\
\hline
\end{tabular}

Table 5: Issue accuracy metrics

\begin{tabular}{lrrrr}
\hline & $\mathrm{P}$ & $\mathrm{R}$ & $\mathrm{F} 1$ & $\mathrm{~N}$ \\
\hline 0: Domestic government & 0.80 & 0.92 & 0.86 & 1097 \\
1: Foreign government & 0.78 & 0.74 & 0.76 & 348 \\
2: Individual & 0.53 & 0.09 & 0.15 & 56 \\
3: Intergovernmental organization & 0.81 & 0.60 & 0.68 & 99 \\
4: Private/business & 0.80 & 0.73 & 0.76 & 233 \\
5: University/school & 0.42 & 0.19 & 0.26 & 37 \\
6: _none_ & 0.00 & 0.00 & 0.00 & 24 \\
\hline
\end{tabular}

Table 6: Target metrics 


\begin{tabular}{rrrrrrrrrrrr}
\hline & $0^{\prime}$ & $1^{\prime}$ & $2^{\prime}$ & $3^{\prime}$ & $4^{\prime}$ & $5^{\prime}$ & $6^{\prime}$ & $7^{\prime}$ & $8^{\prime}$ & $9^{\prime}$ & $10^{\prime}$ \\
\hline 0 & 3 & 0 & 0 & 1 & 0 & 35 & 0 & 1 & 5 & 1 & 0 \\
1 & 0 & 19 & 0 & 1 & 0 & 29 & 0 & 1 & 4 & 0 & 0 \\
2 & 0 & 0 & 32 & 0 & 0 & 17 & 0 & 0 & 3 & 1 & 0 \\
3 & 0 & 0 & 0 & 72 & 0 & 98 & 11 & 2 & 7 & 1 & 0 \\
4 & 0 & 0 & 0 & 2 & 2 & 28 & 0 & 0 & 2 & 1 & 0 \\
5 & 3 & 1 & 1 & 16 & 0 & 752 & 6 & 11 & 17 & 2 & 0 \\
6 & 0 & 0 & 0 & 16 & 0 & 46 & 8 & 0 & 1 & 1 & 0 \\
7 & 1 & 1 & 0 & 1 & 0 & 51 & 0 & 28 & 0 & 0 & 0 \\
8 & 0 & 0 & 0 & 3 & 0 & 37 & 0 & 0 & 233 & 0 & 0 \\
9 & 0 & 0 & 1 & 1 & 1 & 35 & 0 & 1 & 4 & 9 & 0 \\
10 & 0 & 1 & 0 & 0 & 0 & 12 & 0 & 0 & 0 & 0 & 0 \\
\hline
\end{tabular}

Table 7: Form confusion matrix

\begin{tabular}{rrrrrrrrrrrrrrrrrr}
\hline & 0 & $1^{\prime}$ & $2^{\prime}$ & $3^{\prime}$ & $4^{\prime}$ & $5^{\prime}$ & 6 & $7^{\prime}$ & $8^{\prime}$ & $9^{\prime}$ & $10^{\prime}$ & $11^{\prime}$ & $12^{\prime}$ & $13^{\prime}$ & $14^{\prime}$ & $15^{\prime}$ & $16^{\prime}$ \\
\hline 0 & 30 & 0 & 0 & 0 & 0 & 0 & 0 & 0 & 0 & 0 & 0 & 0 & 0 & 0 & 0 & 0 & 0 \\
1 & 0 & 35 & 4 & 0 & 1 & 2 & 4 & 0 & 7 & 3 & 0 & 0 & 1 & 4 & 4 & 1 & 1 \\
2 & 1 & 5 & 92 & 1 & 5 & 2 & 3 & 3 & 3 & 1 & 0 & 1 & 3 & 4 & 5 & 2 & 1 \\
3 & 0 & 0 & 7 & 9 & 0 & 3 & 3 & 0 & 1 & 3 & 0 & 3 & 4 & 4 & 2 & 1 & 3 \\
4 & 0 & 2 & 6 & 2 & 55 & 6 & 2 & 2 & 3 & 3 & 0 & 1 & 1 & 3 & 2 & 3 & 5 \\
5 & 0 & 3 & 4 & 0 & 3 & 125 & 1 & 3 & 1 & 0 & 2 & 4 & 17 & 3 & 2 & 1 & 3 \\
6 & 0 & 4 & 6 & 0 & 1 & 4 & 72 & 3 & 0 & 1 & 0 & 29 & 4 & 1 & 0 & 5 & 0 \\
7 & 0 & 0 & 2 & 0 & 1 & 1 & 1 & 58 & 0 & 0 & 0 & 0 & 0 & 0 & 0 & 0 & 1 \\
8 & 0 & 12 & 7 & 0 & 3 & 4 & 3 & 3 & 30 & 3 & 0 & 5 & 0 & 5 & 4 & 0 & 3 \\
9 & 0 & 1 & 4 & 1 & 9 & 7 & 4 & 1 & 3 & 21 & 4 & 4 & 4 & 6 & 1 & 4 & 4 \\
10 & 0 & 1 & 0 & 0 & 0 & 1 & 0 & 0 & 1 & 2 & 30 & 2 & 0 & 0 & 0 & 0 & 0 \\
11 & 0 & 0 & 2 & 0 & 0 & 2 & 14 & 1 & 1 & 0 & 0 & 314 & 2 & 3 & 0 & 2 & 0 \\
12 & 0 & 0 & 4 & 0 & 1 & 25 & 0 & 0 & 1 & 3 & 0 & 5 & 45 & 3 & 2 & 0 & 5 \\
13 & 0 & 1 & 2 & 1 & 2 & 3 & 4 & 2 & 6 & 2 & 0 & 3 & 1 & 49 & 1 & 0 & 4 \\
14 & 0 & 4 & 3 & 1 & 1 & 1 & 0 & 1 & 1 & 0 & 0 & 1 & 0 & 3 & 99 & 0 & 0 \\
15 & 0 & 0 & 1 & 0 & 2 & 2 & 3 & 1 & 1 & 0 & 0 & 13 & 2 & 2 & 0 & 20 & 5 \\
16 & 1 & 5 & 8 & 2 & 2 & 6 & 4 & 5 & 4 & 1 & 1 & 10 & 4 & 13 & 0 & 5 & 14 \\
\hline
\end{tabular}

Table 8: Issue confusion matrix

\begin{tabular}{rrrrrrrr}
\hline & 0 & $1^{\prime}$ & $2^{\prime}$ & $3^{\prime}$ & $4^{\prime}$ & 5 & 6 \\
\hline 0 & 1005 & 47 & 2 & 3 & 36 & 4 & 0 \\
1 & 77 & 259 & 0 & 9 & 3 & 0 & 0 \\
2 & 46 & 3 & 5 & 0 & 0 & 2 & 0 \\
3 & 22 & 16 & 0 & 59 & 1 & 1 & 0 \\
4 & 54 & 4 & 2 & 2 & 170 & 1 & 0 \\
5 & 26 & 0 & 1 & 0 & 3 & 7 & 0 \\
6 & 19 & 3 & 0 & 0 & 1 & 1 & 0 \\
\hline
\end{tabular}

Table 9: Target confusion matrix 
the confusion matrices for each of the variables. A confusion matrix displays the predicted class of the document compared to its actual class. The column names are marked $x^{\prime}$ to denote the cases which were predicted as class $x$. The rows are the actual class. The value on the diagonal is the number of documents which were classified correctly. So for instance, in table 7 , the value in row 5 (rally/demonstration) and column 7' (riot) is 11, which means 11 articles human coders labeled as rally/demonstration were coded as riots by the classifier. I will discuss each of the closed-ended variables in turn.

For form, $F_{1}$ ranges from 0.11 to 0.85 for all non-none categories. Only three classes have an $F_{1}$ over 0.5 : hunger strike, rally/demonstration and strike/walkout/lockout. It is not for want of training data either, since march, with 191 cases, has an F-score of 0.47. In the confusion matrix, the most noticeable thing is that misclassification of events towards 5', rally/demonstration. The form classifier does not do well to distinguish between the rally and other types of events. All-in-all, the classifier mislabels 342 events as rallies (not including the compound category, rally/demonstrationmarch). On the other hand, few events are mislabeled as a strike/walkout/lockout, the second-most populous category.

What explains this classification error? It may be the case that since rally is the overwhelming form of protest event, everything is very much tinged with the same kind of language. Frequently, when there is an occupation, a boycott, or a march, it is accompanied by a rally. This result is verified by DoCA, where rallies occur in at least $21 \%$ of events 10 . The intention behind creating compound variables is to capture the co-occurrence of forms. But even with that, it doesn't seem like an automated method is able to distinguish between more nuanced types of contentious action, save the hunger strike and the labor strike. But as I will note below, these rates of error seem similar to that of human coders.

For issue, $F_{1}$ ranges from 0.30 to 0.97 for all non-none categories. There are several categories with an F-score above 0.8: abortion, immigration, labor \& work, and religion. Notably, abortion is retrieved with perfect recall and near perfect precision with the minimum number of cases allowed for inclusion (30). Several more classes have F-scores equal or above 0.6: anti-war/peace, criminal justice system, democratization, and environmental. Below that are three are in the 0.5 decile: anti-colonial/political independence, economy/inequality, and racial/ethnic rights. The last

\footnotetext{
${ }^{10}$ http://web.stanford.edu/group/collectiveaction/cgi-bin/drupal/node/17
} 


\begin{tabular}{lrrr}
\hline & $\mathrm{P}$ & $\mathrm{R}$ & $\mathrm{F} 1$ \\
\hline Form & 0.68 & 0.69 & 0.64 \\
Issue & 0.64 & 0.65 & 0.63 \\
Target & 0.77 & 0.79 & 0.77 \\
\hline
\end{tabular}

Table 10: Weighted accuracy metrics for all closed-ended variables.

categories are below 0.5: civic violence, foreign policy, human and civil rights, political corruption/malfeasance, and social services \& welfare.

On the whole, errors aren't as biased towards any one category as they are in the case of protest forms. In the confusion matrix, no single category seems to be driving misclassification. Select pairwise misclassifications seem to be driving the error. Articles labeled economy/inequality are most frequently misclassified as labor \& work (29), which makes substantive sense. Articles labeled as democratization are most frequently misclassified as political corruption/malfeasance (17) and vice versa (25). This seems to follow from the logic that many democratization movements are often driven or at least in response to political corruption by regime elites. But otherwise, there is no single category towards which the classifier exhibits a systematic misclassification.

Lastly, for target, $F_{1}$ ranges from 0.15 to 0.86 for non-none variables. Domestic government, foreign government, intergovernmental organization, and private/business all have F-scores above 0.65. Below that, university/school has a poor F-score of 0.26 and individual has a very poor F-score at 0.15 . In the confusion matrix, we see the same systematic misclassification towards domestic government for all categories: 244 articles are misclassified as such. Like rally/demonstration, this represents a bias towards the targets of protest overall. DoCA again validates this result: more than $51 \%$ of events in DoCA are targeted towards the domestic state.

Table 10 displays the weighted average of accuracy metrics for all closed-ended variables, weighted by the number of cases within each label. Target has the highest $F_{1}$ at 0.77 , driven mostly by the very large number of cases which are correctly classified as domestic government. Form has an $F_{1}$ of 0.64 , mostly driven by the prevalence of the rally/demonstration. Issues has an F-score which is marginally worse (0.63), but as noted in the tables above, the classifier does a reasonably well job given the number and heterogeneity of types of issues.

On the whole, these results are promising. While not perfect, the classification performs reasonably well for the task as hand. One outstanding question is whether these classifiers would perform 


\begin{tabular}{lrr}
\hline & $\mathrm{r}$ & $\mathrm{p}$ \\
\hline Form & 0.513 & 0.158 \\
Issue & 0.794 & 0.000 \\
Target & 0.896 & 0.006 \\
\hline
\end{tabular}

Table 11: Pearson correlation and p-values for coder agreement index and MPEDS $F_{1}$.

as well as human coders at the same task. One virtue of MPEDS which makes it preferable to that of human coders is how we can code articles at scale. If MPEDS's mistakes are similar to those of human coders, then we could still use it with similar confidence and with significantly more speed.

In the second-pass coding process, most if not all articles were coded by more than two coders. Because of this, we can compare the systematic disagreements of human coders with the disagreements between MPEDS and filtered ground-truth values. This may indicate that the machine learning algorithm is doing about as well as human coders, a claim (King and Lowe, 2003) note in their automated event data system.

As a rough test of this, for all articles which had two or more coders, I generated an index which captures coder agreement. For total agreement between coders, the article receives a score of 1 for that variable. If coders have partial agreement, then the article receives a score of 0.5 . If there is total disagreement, the article receives a score of 0 . I summed scores and normalized them by the number of times each value was selected by a human coder. Like in the training data, I excluded those which values which do not appear at least 30 times. I then generated Pearson correlation coefficients between this coder agreement index and the $F_{1}$-score of the variables, as well as their corresponding p-values. These values are reported in Table 11.

The claim that these classifiers are doing as well as human coders would seem to hold for the issue and target variables. Issue has a correlation of 0.794 with $p<0.001$ and target has a correlation of 0.896 with a $p<0.01$. For form, however, the correlation is 0.513 , but the p-value does not meet the 0.05 criteria for statistical significance. This provides us with more evidence that we can use these classifiers with confidence as a replacement for human coders. 


\section{$5 \quad$ Open-ended coding}

While the haystack and closed-ended coding tasks classify the article from sets of known categories, the process of extracting text from the document which relate to a variable of interest involves identification of relevant entities and parsing correct information from candidate text snippets.

MPEDS focuses on three open-ended variables of interest: size, social movement organization, and location. Size is important as a metric of protest intensity and has been noted to be a predictor of news bias (Oliver and Maney, 2000). More recently, Biggs (2016) has noted that getting protest intensity correct in protest event datasets is more theoretically important than getting frequency counts right. Thus size is an important variable to code and code correctly. Social movement organizations are significant insofar as the large and still growing literature on the importance of movement organizations, initially motivated by the resource mobilization perspective (McCarthy and Zald, 1977: Zald and McCarthy, 1979) and continuing throughout the current focuses in movement scholarship. Since the establishment in resource mobilization, the focus on social movement organizations has gained insights from organization theory (Davis et al., 2005) and become a standard category for protest event analysis. Obtaining location is simply necessary for us to locate a protest event in space. If there is a date, form, and issue for an event, it does not do us well unless we know where it is.

Although each of these can be extracted from the same text, automating the extraction of each variable has its own set of challenges. There is a significant subset of machine learning research which focuses on labeling sequences (known as sequence classification), such as genomic data and sequences of strings (Xing, Pei and Keogh, 2010). Within natural language processing, the process of identifying entities in text is known as named-entity recognition (NER). Several popular tools have been made to accomplish NER, including Stanford CoreNLP (Finkel, Grenager and Manning, 2005) and MALLET (Sutton and McCallum, 2006). Most modern NER systems use some sort of probabilistic model for text classification. For both Stanford CoreNLP and MALLET, those are conditional random fields (CRF) and hidden Markov models (HMM). Although theoretically these tools could classify any annotated text for a snip of specific text, the most common NER classifiers have been trained to find three entities: organization, person, and location. Luckily, that is two of the three variables we're interested in. Unluckily, however, additional work is needed to 
distinguish between a social movement organization and any other kind of organization, including organizations the protest may be opposing. Similarly, we need to be able to distinguish between locations in which the protest has occurred and other locations which appear in the article.

Size presents another host of issues. Although numbers and numerical words are relatively easy to find within texts, separating out numbers which represent protest size estimates from other numbers is more difficult. In a typically news article on a protest event, numbers may include not only crowd size, but also numbers arrested, injured, and killed, and crowd size estimates from a protest occurring at an earlier point in time. To complicate matters, estimating crowd size itself is not a very exact science and is contested depending on the social position of the person reporting the number relative to the protest (McPhail and McCarthy, 2004). This contestation often plays out within the news article itself with writers often reporting both organizer and police estimates. Each of these variables requires a particular set of rules for extraction. Below I describe methods of size, organization, and location extraction.

Size. The pseudocode in Figure 4 outlines the steps behind detecting protest event sizes. In words: the algorithm anchors the size in a set of digits or number word, then infers if it is a protest size by the context around it. There is often a word which denotes a subject after the numerical term. Some of these are dead giveaways (protester, activist) but most of the time they are other, more general words which refer to a kind of individual (e.g. people, mothers, Egyptians). I created a regular expression to capture a wide swath of these terms and also created a comprehensive list of ethnicities and nationalities from Wikipedia 11 . Before the numerical word may be another number which is a descriptor (e.g. hundreds of thousands). If no subject word had been identified after the word in question, a word occurring before the number may denote a size (e.g. crowd of 10,000). I then return the all the sizes found in the article. Sizes are then resolved to a numerical estimation of the prose description. This estimation is intended to represent scale rather than exact amounts. For instance, "tens of thousands" turns into 10,000, "dozens" turns into 10, and "several" turns into 2.

The previous metrics of precision, recall, and F-score are not appropriate because of the resolution into a continuous variable, thus size accuracy is evaluated both by finding exact matches and

\footnotetext{
11 "List of contemporary ethnic groups" https://en.wikipedia.org/wiki/List_of_contemporary_ethnic_groups and "List of people by nationality" https://en.wikipedia.org/wiki/Lists_of_people_by_nationality
} 


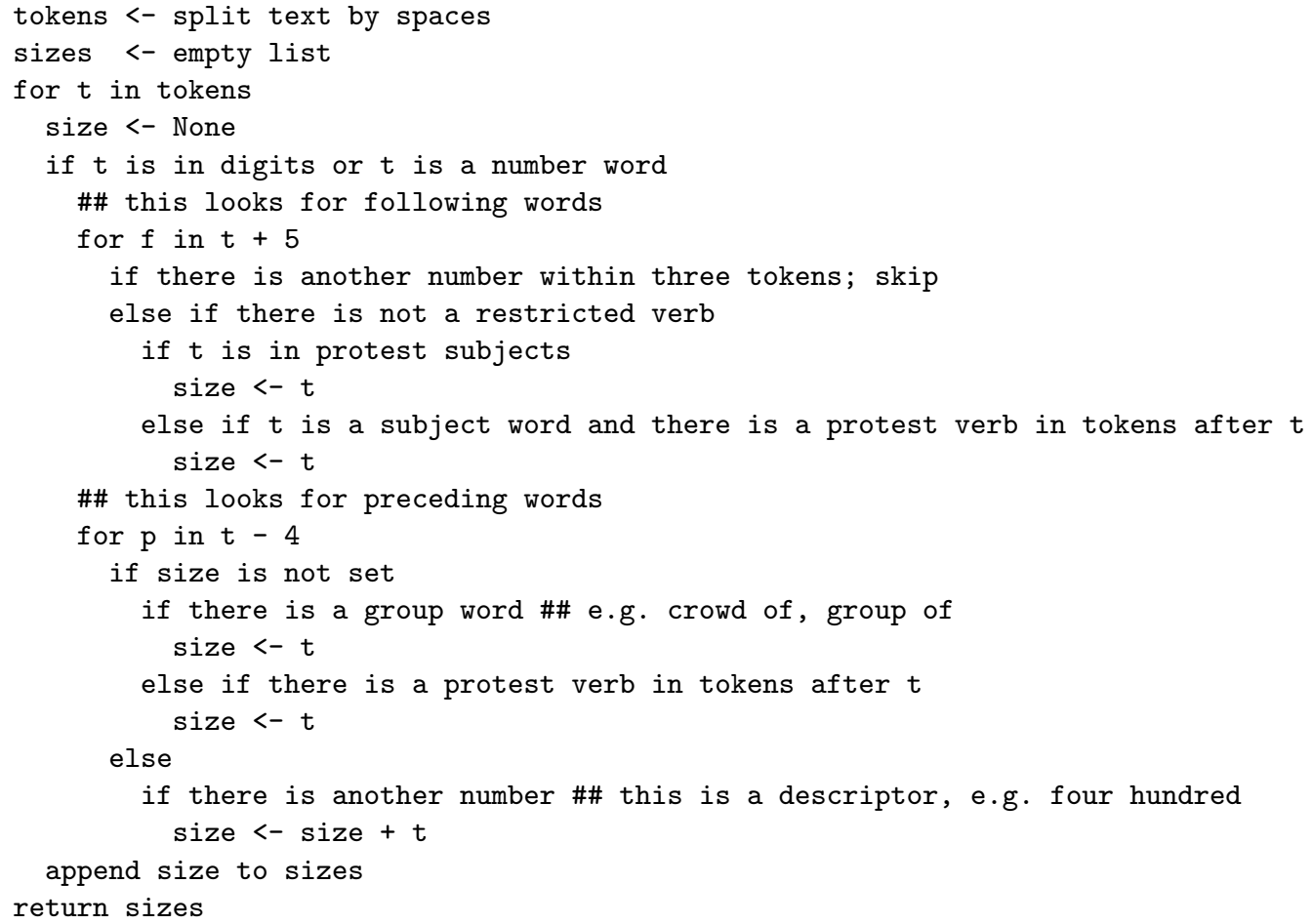

Figure 4: Size extraction algorithm in pseudocode. 


\begin{tabular}{lrrrrr}
\hline \# values & Non-none & \# Equal & Equal proportion & Mean Jaccard index & Total \\
\hline 1 & & & & & \\
2 & 921 & 513 & 0.51 & 0.56 & 999 \\
3 & 921 & 280 & 0.28 & 0.49 & 999 \\
\hline
\end{tabular}

Table 12: Size accuracy

calculating the mean Jaccard index across articles. The Jaccard index is calculated by taking the intersection of the set of values identified by a human coder and the set of values identified by the machine, and dividing the number of intersections with the union of those two sets. I calculated the Jaccard index with the intent of providing some notion of partial agreement between the human coded and machine coded sets. Given that the model is fixed and not retrained dynamically, no cross-validation was performed. I tested taking the highest $k$ sizes in the document for $k=1,2,3$. I also calculated how many articles had the exact same size as reported by human coders. The results are presented in Table 12 . The best results are when we report one size for each article: over half of the variables were equal to their hand coded values and the Jaccard index is at 0.55. Taking the top index is bound to create some false positives (for instance, an article reports a march that occurred in the past which trumped the size of the current event), and it may make sense instead to report the top two values instead of only one. Given that we don't see much of a decrease in the Jaccard index when two values are returned, this may be a better strategy for data production.

Overall, this is somewhat of a brute force method of extracting size, and there may be some more sophisticated methods for extracting size. One method would involve training an NER model to look explicitly for protest event size. Another could be to do a shallow parsing of sentences with a toolkit like Stanford CoreNLP and more explicitly identify subject-verb-object triads which look like protest size estimates. But for the time being, this represents a better solution than identifying numerical words in isolation.

Location: I used the CLIFF Entity Extractor created by the MIT Center for Civic Media ${ }^{2}$ to identify location information. CLIFF identifies all sequences of text in the three classes used in the CoNLL 2003 shared task in named-entity recognition (Tjong Kim Sang and De Meulder, 2003): location, person, and organization, using the Stanford CoreNLP named-entity recognition

\footnotetext{
12 https://github.com/c4fcm/CLIFF
} 
classifier. Building on this, CLIFF enriches the location data by attempting to infer the "focus" of a news article. It combines this information with a geoparser called CLAVIN 13 . CLAVIN indexes the popular GeoNames gazetter, which contains several million geographical entries. CLIFF identifies organizations and provides comprehensive additional location information based on GeoNames, such as administrative districts, FIPS codes, latitude, and longitude. Accordingly, MPEDS returns up to four locations, their latitudes and longitudes from the source text, as well as the same information from the dateline.

In this paper, I offer no accuracy results for location because it is not clear what the best metric of this may be. While precision and recall metrics may make sense, the resolution provided by CLIFF may make it possible to estimate deviations from latitude and longitude from the human coded values. That itself may be more informative than just getting the actual text correct.

Social movement organizations. I use a custom-trained CoreNLP NER classifier to detect social movement organizations in the text. The out-of-the-box classifier for CoreNLP uses the training data from the CoNLL 2003 shared task. However, using this classifier yields a very high number of false positives, that is, a high number of organizations identified which are decidedly not social movement organizations. In an unaltered run of over many news texts, organizations include senate, congress, supreme court, department of defense, walmart and monsanto. These organizations are typically the target of protests and need to be distinguished from movement organizations.

I compared three different classifiers before settling on the newly-trained NER model. I first used a dictionary-based system in order to match social movement organizations. For that purpose, I integrated two organization dictionaries. The first is a cleaned set of organizations drawn from DoCA 4 . The second is a modified list drawn from the Encyclopedia of Organizations database (also called Associations Unlimited and thus abbreviated as AU 45 I collected all organization names in the Public Affairs, Labor, Social Welfare, and Environmental categories at the time of writing. Further efforts to expand this database could retrieve the historical lists back to 1961. This task would take some effort to digitize paper records for years before 1995.

These systems, however, produced poor results, as noted in Table $13, F_{1}$ for the Associated

\footnotetext{
$\sqrt[13]{\text { https://clavin.bericotechnologies.com/ }}$

${ }^{14}$ The Appendix in Wang and Soule (2012) report the process of matching and cleaning these data. Thanks to the authors for providing them.

15 http://find.galegroup.com/gdl/help/GDLeDirEAHelp.html
} 
Unlimited database is 0.16 , and it is 0.18 for DoCA. There are several possible hypotheses for this discrepancy. These databases tend to be US-centric, DoCA by design and AU by bias. DoCA excludes all events which do not take place in the US. For those categories which I collected from the AU database, 27,835 (21,568 regional and 6,267 national) organizations are based in the US compared to 6,481 based outside of the US. In addition, AU organizations had to be formally constituted, usually with a phone number and contact information, whereas many social movement organizations are briefly lived and not available for contact. Therefore it seemed to be more adequate to rely on an NER tool based on MPEDS-produced training data. In the future, however, DoCA could possibly be used as additional training data if their mentions can be matched to their source article texts.

I compare all the classifiers in Table 13 using random permutation cross-validation. I shuffled the dataset into a training and test set with a 90/10 split over three iterations and took the average of the metrics. The baseline classifier trained on CoNLL data yields an F-score of 0.32. There is high recall, given the inclusion of a wide variety of organizations, but the precision is 0.21 , which is far too low for use in MPEDS. The classifier trained on MPEDS data, however, reports precision of 0.71 , recall of 0.49 , and F-score of 0.58 , which is a dramatic improvement over the other solutions.

Future directions in extracting movement organizations could involve several more complicated steps. The solution of shallow parsing mentioned earlier in the size extraction subtask could be applied here. Furthermore, more dictionaries could be incorporated, such as the CAMEO and PETRARCH actor ontologie: $\$$, and new dictionaries could be developed with an eye towards intrastate protest and conflict. This paper also does not explore the possibly of ensemble methods, such as supplementing the MPEDS-trained NER classifier and allowing the dictionaries a (weighted) vote on each word in the article.

The open-ended coding task is the least developed part of the MPEDS system and at the same time one of the hardest. Extracting unstructured text from a set of news articles with heterogeneous origins is a difficult task. Size, location, and organizations are mentioned in a number of different ways and are embedded in a variety of different grammatical structures. However, given the evaluation presented here, it seems very hopeful that much more progress can be made

\footnotetext{
${ }^{16}$ https://github.com/openeventdata/petrarch/blob/master/petrarch/data/dictionaries/Phoenix. agents.txt and https://github.com/openeventdata/petrarch/blob/master/petrarch/data/dictionaries/ Phoenix.MilNonState.actors.txt
} 


\begin{tabular}{lccc}
\hline & $\mathrm{P}$ & $\mathrm{R}$ & $\mathrm{F} 1$ \\
Classifier & & & \\
\hline au & 0.55 & 0.10 & 0.16 \\
doca & 0.33 & 0.13 & 0.18 \\
baseline & 0.21 & 0.71 & 0.32 \\
mpeds & 0.72 & 0.49 & 0.58 \\
\hline
\end{tabular}

Table 13: Social movement organization accuracy

and open-ended data can be extracted with a high level of accuracy. Several possible alternative solutions have been noted above. More are bound to be developed by engaging with the cutting edge in sub-areas of computer science and natural language processing, including new sequence classification models, and fast and accurate sentence parsing such as dependency parsing.

\section{Discussion}

The results presented here provide promise of rich event data for protest events with significantly less human intervention. They provide much more information than the sparse, dyadic datasets which are popular in the literatures in conflict forecasting and international relations. They also point to important improvements to current practice for generating event data for social movement scholarship. The advantage of time saved in generating data is the most important improvement. Once a relatively small amount of training data has been created, and classifiers have been created and validated, the actual data generation process involves the simple steps of apply the system to a corpus of news articles of interest. The training data produced by human coders by the MPEDS project amounted to some 20,000 unique articles, with nearly 3,000 positive news articles filtered for second pass coding. We also coded the articles more than one time to ensure reliability. With the MPEDS annotation interface, this work took less than two years with between 5 to 8 coders working at once. With these classifiers, we can produce protest event data from the whole of a large corpus, say the Gigaword corpus of 10 million newswire stories, in a matter of minutes. Compare this process to the decade-long task of producing the data for the Dynamics of Collective Action project, in which articles were coded only once and thus introduce more room for error.

The room for error is evident in the manual inspection of MPEDS's coding of DoCA data described above, in which 31 of 50 articles were "false false positives". DoCA is considered to be 
the state-of-the-art for protest event data in the US in the field of social movement research. It represents the largest US-based effort to collect a comprehensive account of protest events over its three and a half decades. This dataset of 21 thousand events, however, may not be complete and may misrepresent the true population of events which the New York Times covered over this time period. Over a dozen articles and at least one book have been produced using DoCA data. It would be a fruitful exercise to interrogate the results of this scholarship based on the data produced by MPEDS using DoCA's parameters.

That said, MPEDS as presented here is far from ideal. There are several areas which would be fertile ground for improvement. They are presented here from the most fundamentally omissions to the least.

Multiple events in a single article. While MPEDS excels at finding news articles mentioning social protest and conflict, it is only limited to addressing one event per article. A single news article may mention multiple events, for instance, a set of coordinated events occurring across different locations. This is where systems such as PETRARCH shine because they attempt to collect event data on the sentence level. Ideally, an a human-annotated training set would include information down to the paragraph and sentence-level in order to describe where information on the protest event can be found. Within the MPEDS project we have been able to collect these data into our hand coding system but have not yet implemented it into the classification process. If we are successful, it will allow us not only to identify multiple events in text, but to more accurately classify particular kinds of protest (either by form, issue, or target) with its appropriate textual components. Marakov et al. (2015) suggest using "anchor" words to identify protests at a sentenceor paragraph-level and merging them up into separable units. But their solution has not yielded results which are better than the results presented here.

Deduplication. Currently, MPEDS does not contain any mechanism for removing multiple mentions of a single event. This problem becomes more dire with the introduction of multiple news sources. This is a task within computer science known as record linkage ${ }^{17}$. Some of the fields produced by an MPEDS record may contribute to linking records together more accurately than others. But this is not addressed by the current version of the system.

Time-shifting. The system assumes that the event takes place on the same day (or at most,

\footnotetext{
17 https://en.wikipedia.org/wiki/Record_linkage
} 
the day before) the reporting date. While this is often the case, it is not a guarantee. A possible solution would be to use a time annotation library such as SUTime (Chang and Manning, 2012) and perform an estimated time shift to more accurately report the date of the event.

Multiple languages. MPEDS is restricted to coding only English-language articles. This obviously introduces a Western (and moreover anglophone) bias into the events which are reported. However, cross-linguistic classification and information extraction is a large and growing area of natural language processing. Additionally, other than the size extraction subtask and dictionaries available for organizations, producing new training data and generating new classifiers based on non-English languages is well within reason for the current machinery used to produce the MPEDS system.

Single closed-ended value. In the current version of MPEDS, the closed-ended variable can only take on one value at a time. While the MPEDS annotation interface allows for compound solutions (e.g. anti-war/peace and human rights), in practice these compounds are not robust enough across coders to make it into our final analysis. However, this is not a fundamental error. Instead of a single value, MPEDS could produce multiple confidence estimates for each variable. This may be skewed towards one category in which there is a high amount of confidence (e.g. abortion) but be useful for categories which have a significant amount of overlap (e.g. democratization and political corruption/malfeasance).

Temporal changes in language. Lastly, language surrounding protest can and does change over time. The history of movement scholarship itself teaches us that the rhetoric around movement activity once took the character of a "maddening crowd." Similarly, editorial and stylebook decisions can fundamentally change how a news source talks about an event. For instance, the virulent homophobia of former New York Times editor A. M. Rosenthal prevented the word gay from appearing in the Times (without scare quotes) until after his editorship ended, in 1986 (Elliot, 2014, Kaiser, 2012).

Most of these changes are not insurmountable, but they will take effort to extend the version of MPEDS presenting in this paper. All aspects of MPEDS are intended to be openly released and extendable by social and computational scientists. Currently, only the annotation interface of MPEDS has been released as open-source (https://github.com/alexhanna/mpeds-coder). However, by fall 2016 the whole MPEDS pipeline will be released as open-source software, most 
likely as a virtual machine package which encapsulates all of its necessary components.

\section{Conclusion}

This paper advances the state of protest event data generation in the social science using methods imported from computer science and statistics. Protest event data is a critical component in a number of subfields in sociology, political science, and other social sciences. Given the increasing availability of various electronic sources and the advances in natural language processing for large text sources, using automated methods is a natural move for social movements research and is essential for tasks like political instability monitoring, which requires real-time or near real-time data. The MPEDS project is innovative in combining machine learning approaches with deep substantive knowledge of the problems of identifying and coding collective events in news sources. MPEDS aims to be more efficient, replicable, and usable than the many of the current tools in social movements and political science. In addition, the data it produces will be rich enough for use in social movement research.

Even if many of the technical challenges presented here are resolved, many challenges will remain in the generation of new protest event data. First and foremost, the well-known biases of newspapers will not go away. Newspapers are businesses and as the institution of the news changes and consolidates towards less ownership, less diverse news may be a consequence. MPEDS cannot solve the consolidation and selection biases of news institutions, but it can allow for easier coding of new and more varied news sources. Additionally, reliance on news reports has forced researchers interested in generating event data to gather news articles from document databases such as Lexis-Nexis and Factiva. Although it may be easier to gather data in real-time for new events using web scrapers and RSS feeds, historical data is more difficult. These databases are typically only available to libraries which have purchased access, and even then they set up significant obstacles to downloading news articles at scale. The legal status of using news articles in event data research is thorny (Schrodt, 2014), and these databases understandably take an aggressive posture to limit access to the intellectual property they store. There may, however, be alternatives to this model of access for researchers. The Linguistic Data Consortium, for instance, provides free access to text repositories to member institutions. And the Open Event Data Alliance (http: 
//openeventdata.org) is an attempt to develop similar infrastructure for event data researchers.

Institutional barriers notwithstanding, systems such as MPEDS have the potential to change the way we study social movements. They can allow us to ask questions and test hypotheses in ways which we couldn't have imagined in a prior era. For example, by generating protest event data in real-time, it may be possible to test hypotheses about mobilization in real-time through political forecasting, that is, making claims about events or trends in events which have not happened yet. Forecasting in social science can be used as a means of testing competing theories. Furthermore, advances in protest event data can allow for social movement theory to be updated in light of massive changes in communication and informational technologies, the constantly-evolving forms of social movement organizations and fields, and new movements against capitalism, authoritarianism, and racism, as well as contention stemming from right-wing nativist and anti-state movements. These measurement tools make possible the ground on which new social science theory can be built.

\section{A Definition of an MPEDS event}

For this project, a protest event should be coded "yes" if there is sufficient information to identify a discrete protest event that occurred in the past or is being planned for the future. A protest mention should include information about the time and location of the event and the actors or claims involved. More specifically a protest mention should be an event that involves:

1. Location of the protest and date that the event occurred

(a) Protest can be occurring, have occurred in the past, or is being planned for the future

2. A claims-making or grievance expression. There is a message that is attempting to influence social change, policies, or actions by major institutions. The message can be advocating for or opposing policies advocated by others.

3. Some of the actors must not be government officials or institutional leaders like university officials or business owners.

(a) An actor does an action or makes a claim; merely being in the audience does not count.

(b) Actions by leaders of organizations whose purpose is to advocate for social change goals ARE protest actions. This is the difference between, say, the Chancellor of the University and the head of the National Organization for Women.

(c) We will leave in actions initiated by unions if they involve claims-making or expression of grievance.

List of acceptable protest events:

- Rally, strike, protest, march, demonstration, vigil, picketing, leafleting, occupation or sitin, blockade/slowdown/disruption, strike/walkout/lockout, civil disobedience, boycott, riot, property damage, symbolic display, press conference or news conference by a non-governmental 
organization (NOT an elected official, NOT government agency, NOT candidate for office), commemoration, counter-protest, walk-out, sit-in, ballot events, online protest (has to meet same criteria as offline protest).

- Routine events by social movements groups (e.g. gay pride parade).

- It is likely that there will be other forms of protest (e.g. reading a banned book, chaining oneself to a desk, carrying a mattress). These can be included but must be connected to larger claims-making movements.

List of NOT acceptable protest events:

- Individual grievances, crimes, and public displays of anger that are not expressive of either social goals or the shared grievances of a social category/group.

- Isolated crimes, lawsuits, court cases, policy decisions that do not include expressions of a broader group or collective.

- Political campaign events.

- Violence or vandalism unrelated to a broader claim or issue; the act of violence is primary form of protest (e.g. bombing of Sterling Hall).

- Press conference or news conference by a governmental organization (elected official, government agency, candidate for office).

- "Routine" politics, political campaigns, speeches by elected officials or those seeking electoral office.

- Interviews and speeches by celebrities.

- Simple charity activity (collect money, no policy demands).

- Entirely personal actions (e.g. crime, personal expression), self-expression (purpose of gathering is entirely inward, not outward). A party or religious ritual as an end in itself is NOT a protest event, but these forms could be protest events if the stated purpose is to make a claim, express a grievance, or advocate for/against some policy change.

- Acts of terrorism - a rough definition of terrorism as an event aimed at intentionally destroying large amounts of property or injuring people.

- Selma rule: exclude coverage of historical protests that are not happening contemporaneously; we only want articles that discuss protest events that are directly relevant at the time of writing, at least within a year of the reporting date.

\section{B Search string for MPEDS articles}

We use a search string to collect data from Lexis-Nexis and to filter out articles in the New York Times Annotated Corpus and the Annotated Gigaword collection. Our intent with this string is full recall without regard to precision. Therefore the string is as inclusive as possible. This was constructed by referring to existing movement search research (Maney and Oliver, 2001; Strawn, 2010). In our testing, we were able to retrieve all DoCA articles over periods of highest event density. 
boycott* OR press conference OR news conference OR protest* OR strik* OR rally OR ralli* OR riot* OR sit-in OR occupation OR mobiliz* OR blockage OR demonstrat* OR marchi* OR marche* NOT protestant*

\section{Software - Chapter}

Article data were processed using Python version 2.7.10, pandas version 0.17.1 (McKinney, 2010) and numpy version 1.10.4 (van der Walt, Colbert and Varoquaux, 2011). MPEDS was built with scikit-learn version 0.17 (Pedregosa et al., 2011), Stanford NER v3.6.0 (Finkel, Grenager and Manning, 2005), and CLIFF (https://github.com/c4fcm/CLIFF). Visualization and correlational analysis was performed with the $\mathrm{R}$ statistical package, version 3.2.3 (R Core Team, 2015). Graphs were produced with ggplot2, version 2.1.0 (Wickham, 2009).

\section{References}

Beissinger, Mark R. 2002. Nationalist Mobilization and the Collapse of the Soviet State. Cambridge University Press.

Biggs, Michael. 2016. "Size Matters: Quantifying Protest by Counting Participants." Sociological Methods 83 Research pp. 1-33.

Blei, David M, Andrew Y Ng and Michael I Jordan. 2003. "Latent dirichlet allocation." The Journal of Machine Learning Research 3:993-1022.

Brandt, Patrick T., John R. Freeman and Philip A. Schrodt. 2011. "Real time, time series forecasting of inter-and intra-state political conflict." Conflict Management and Peace Science 28(1):41-64.

Carter, Gregg Lee. 1983. "Explaining the Severity of the 1960's Black Rioting." Unpublished Dissertation. Columbia University .

Chang, Angel X and Christopher D Manning. 2012. SUTime: A library for recognizing and normalizing time expressions.

Conover, Michael, Jacob Ratkiewicz, Matthew Francisco, Bruno Gonçalves, Filippo Menczer and Alessandro Flammini. 2011. Political Polarization on Twitter. In Proceedings of 5th International Conference on Weblogs and Social Media (ICWSM).

URL: http://www.aaai.org/ocs/index.php/ICWSM/ICWSM11/paper/download/2847/3275

Croicu, Mihai and Nils B Weidmann. 2015. "Improving the selection of news reports for event coding using ensemble classification." Research $\&$ Politics 2(4).

URL: http://rap.sagepub.com/content/2/4/2053168015615596

Davenport, Christian. 2010. Media Bias, Perspective, and State Repression: The Black Panther Party. Cambridge University Press.

Davis, Gerald F, Doug McAdam, W Richard Scott and Mayer N Zald. 2005. Social movements and organization theory. Cambridge University Press.

Earl, Jennifer, Andrew Martin, John D. McCarthy and Sarah Soule. 2004. "The Use of Newspaper Data in the Study of Collective Action." Annual Review of Sociology 30:65-80. 
Earl, Jennifer and Sarah A. Soule. 2006. "Seeing blue: A police-centered explanation of protest policing." Mobilization: An International Quarterly 11(2):145-164.

Earl, Jennifer, Sarah A. Soule and John D. McCarthy. 2003. "Protest under fire? Explaining the policing of protest." American Sociological Review 68(4):581-606.

Elliot, Thomas. 2014. The Cultural Consequences of Social Movements: The LGBT Movement and the Transformation of Discourse About Homosexuality. In Paper presented at the Young Scholars in Social Movements Conference. Notre Dame, IN.

Finkel, Jenny Rose, Trond Grenager and Christopher Manning. 2005. Incorporating non-local information into information extraction systems by gibbs sampling. In Proceedings of the $43 \mathrm{rd}$ Annual Meeting on Association for Computational Linguistics. Association for Computational Linguistics pp. 363-370.

Franzosi, Roberto. 1987. "The Press as a Source of Socio-Historical Data: Issues in the Methodology of Data Collection from Newspapers." Historical Methods: A Journal of Quantitative and Interdisciplinary History 20(1):5-16.

Franzosi, Roberto. 2004. From Words to Numbers: Narrative, Data, and Social Science. Cambridge University Press.

Franzosi, Roberto. 2010. Quantitative narrative analysis. Vol. 162 Sage.

Gerner, Deborah J. and Philip A. Schrodt. 1994. "Validity assessment of a machine-coded event data set for the Middle East, 1982-92." American Journal of Political Science 38(3):825-854.

Goldstone, Jack A., Robert H. Bates, David L. Epstein, Ted Robert Gurr, Michael B. Lustik, Monty G. Marshall, Jay Ulfelder and Mark Woodward. 2010. "A global model for forecasting political instability." American Journal of Political Science 54(1):190-208.

Grimmer, Justin and Brandon M. Stewart. 2013. "Text as data: The promise and pitfalls of automatic content analysis methods for political texts." Political Analysis pp. 1-31.

Grimmer, Justin, Solomon Messing and Sean J Westwood. 2014. Estimating heterogeneous treatment effects and the effects of heterogeneous treatments with ensemble methods. Technical report Working paper). Stanford, CA: Stanford University. Retrieved from http://stanford. edu/ jgrimmer/het. pdf.

Hopkins, Daniel J and Gary King. 2010. "A method of automated nonparametric content analysis for social science." American Journal of Political Science 54(1):229-247.

Hutter, Swen. 2014. Protest Event Analysis and Its Offspring. In Methodological Practices in Social Movement Research, ed. Donatella Della Porta. Oxford University Press.

Kaiser, Charles. 2012. "When The New York Times Came Out of the Closet." The New York Review of Books .

URL: http://www.nybooks.com/daily/2012/09/25/when-new-york-times-came-out-closet/

Khawaja, Marwan. 1994. "Resource mobilization, hardship, and popular collective action in the West Bank." Social Forces 73(1):191-220. 
King, Gary and Will Lowe. 2003. "An automated information extraction tool for international conflict data with performance as good as human coders: A rare events evaluation design." International Organization 57(03):617-642.

Kriesi, Hanspeter, Ruud Koopmans, Willem Duyvendak and Marco Giugni. 1995. New Social Movements in Western Europe: A Comparative Analysis. University of Minnesota Press.

Leetaru, Kalev and Philip A. Schrodt. 2013. "GDELT: Global data on events, location, and tone, 1979-2012.". Accessed: 2014-01-05.

URL: http://eventdata.psu.edu/papers.dir/ISA.2013.GDELT.pdf

Maney, Gregory M. and Pamela E. Oliver. 2001. "Finding Collective Events Sources, Searches, Timing." Sociological Methods \& Research 30(2):131-169.

Marakov, Peter, Jasmine Lorenzini, Klaus Rothenhäusler and Bruno Wüest. 2015. "Towards automated protest event analysis.". Presented at New Frontiers of Automated Content Analysis in the Social Sciences Conference. University of Zurich, July 1-3.

URL: http://www.aca-zurich-2015.org/files/MakarovEtAl-ACA-Zurich-2015.pdf

McAdam, Doug and Yang Su. 2002. "The war at home: Antiwar protests and congressional voting, 1965 to 1973." American Sociological Review 67(5):696-721.

McCarthy, John D., Clark McPhail and Jackie Smith. 1996. "Images of protest: Dimensions of selection bias in media coverage of Washington demonstrations, 1982 and 1991." American Sociological Review p. 478-499.

McCarthy, John D and Mayer N Zald. 1977. "Resource mobilization and social movements: A partial theory." American journal of sociology pp. 1212-1241.

McKinney, Wes. 2010. Data Structures for Statistical Computing in Python. In Proceedings of the 9th Python in Science Conference, ed. Stéfan van der Walt and Jarrod Millman. pp. 51 - 56.

McPhail, Clark and John McCarthy. 2004. "Who counts and how: estimating the size of protests." Contexts 3(3):12-18.

Mohr, John W. and Petko Bogdanov. 2013. "Topic Models and the Cultural Sciences." Poetics 41:545-569.

Murphy, Kevin P. 2012. Machine learning: a probabilistic perspective. The MIT Press.

Myers, Daniel J. 2000. "The Diffusion of Collective Violence: Infectiousness, Susceptibility, and Mass Media Networks." American Journal of Sociology 106(1):173-208.

Myers, Daniel J. and Beth Schaefer Caniglia. 2004. "All the rioting that's fit to print: Selection effects in national newspaper coverage of civil disorders, 1968-1969." American Sociological Review 69(4):519-543.

URL: http://asr.sagepub.com/content/69/4/519.short

Nardulli, Peter F, Scott L Althaus and Matthew Hayes. 2015. "A Progressive Supervised-learning Approach to Generating Rich Civil Strife Data." Sociological Methodology pp. 1-36.

Oliver, Pamela E and Gregory M Maney. 2000. "Political Processes and Local Newspaper Coverage of Protest Events: From Selection Bias to Triadic Interactions." American Journal of Sociology 106(2):463-505. 
Olzak, Susan. 1989. "Labor unrest, immigration, and ethnic conflict in urban America, 1880-1914." American Journal of Sociology 94(6):1303-1333.

Olzak, Susan. 1992. The Dynamics of Ethnic Competition and Conflict. Stanford University Press.

Ortiz, David G., Daniel J. Myers, Eugene N. Walls and Maria-Elena D. Diaz. 2005. "Where do we stand with newspaper data?" Mobilization: An International Quarterly 10(3):397-419.

Pedregosa, Fabian, Gaël Varoquaux, Alexandre Gramfort, Vincent Michel, Bertrand Thirion, Olivier Grisel, Mathieu Blondel, Peter Prettenhofer, Ron Weiss, Vincent Dubourg et al. 2011. "Scikit-learn: Machine learning in Python." The Journal of Machine Learning Research 12:28252830 .

R Core Team. 2015. R: A Language and Environment for Statistical Computing. Vienna, Austria: $\mathrm{R}$ Foundation for Statistical Computing.

URL: https://www.R-project.org/

Schrodt, Philip A. 2014. The legal status of event data.

URL: https://asecondmouse.wordpress.com/2014/02/14/the-legal-status-of-event-data/

Schrodt, Philip A., Erin M. Simpson and Deborah J. Gerner. 2001. Monitoring conflict using automated coding of newswire reports: a comparison of five geographical regions. In Conference 'Identifying Wars: Systematic Conflict Research and it's Utility in Conflict Resolution and Prevention', Uppsala. Citeseer pp. 8-9.

Schrodt, Philip A., James Yonamine and Benjamin E. Bagozzi. 2013. Data-based Computational Approaches to Forecasting Political Violence. In Handbook of Computational Approaches to Counterterrorism. Springer p. 129-162.

Schrodt, Philip A., John Beieler and Mohammed Idris. 2014. "Three's a Charm?: Open Event Data Coding with EL:DIABLO, PETRARCH, and the Open Event Data Alliance.". Paper presented at the ISA Annual Convention.

Schrodt, Philip A, Shannon G Davis and Judith L Weddle. 1994. "Political science: KEDS-a program for the machine coding of event data." Social Science Computer Review 12(4):561-587.

Soule, Sarah A. 2009. Contention and corporate social responsibility. Cambridge University Press.

Spilerman, Seymour. 1970. "The causes of racial disturbances: A comparison of alternative explanations." American Sociological Review 35:627-649.

Spilerman, Seymour. 1971. "The causes of racial disturbances: Tests of an explanation." American Sociological Review 36:427-442.

Spilerman, Seymour. 1976. "Structural characteristics of cities and the severity of racial disorders." American Sociological Review 41:771-793.

Strawn, Kelley D. 2010. "Protest Records, Data Validity, and the Mexican Media: Development and Assessment of a Keyword Search Protocol." Social Movement Studies 9(1):69-84.

Sutton, Charles and Andrew McCallum. 2006. "An introduction to conditional random fields for relational learning." Introduction to statistical relational learning pp. 93-128. 
Tarrow, Sidney. 1989. Democracy and Disorder: Protest and Politics in Italy, 1965-1975. Oxford University Press.

Tilly, Charles, Louise Tilly and Richard Tilly. 1975. The Rebellious Century: 1830-1930. Harvard University Press.

Tjong Kim Sang, Erik F and Fien De Meulder. 2003. Introduction to the CoNLL-2003 shared task: Language-independent named entity recognition. In Proceedings of the seventh conference on Natural language learning at HLT-NAACL 2003-Volume 4. Association for Computational Linguistics pp. 142-147.

van der Walt, S., S. C. Colbert and G. Varoquaux. 2011. "The NumPy Array: A Structure for Efficient Numerical Computation." Computing in Science Engineering 13(2):22-30.

Wang, Dan J. and Sarah A. Soule. 2012. "Social movement organizational collaboration: Networks of learning and the diffusion of protest tactics, 1960-19951." American Journal of Sociology 117(6):1674-1722.

Wickham, Hadley. 2009. ggplot2: Elegant Graphics for Data Analysis. Springer-Verlag New York. URL: http://ggplot2.org

Woolley, John T. 2000. "Using media-based data in studies of politics." American Journal of Political Science 44(1):156-173.

Wueest, Bruno, Klaus Rothenhäusler and Swen Hutter. 2013. "Using Computational Linguistics to Enhance Protest Event Analysis.".

URL: http://www.bruno-wueest.ch/files/Wueest_Hutter_Rothenhaeusler_2013.pdf

Xing, Zhengzheng, Jian Pei and Eamonn Keogh. 2010. "A brief survey on sequence classification." ACM SIGKDD Explorations Newsletter 12(1):40-48.

Zald, Mayer N and John D McCarthy. 1979. "Social Movement Industries: Competition and Cooperation among Movement Organizations.". 